\title{
Synthesis of the entire carbon framework of the kedarcidin chromophore aglycon $\uparrow$
}

\author{
Fumihiko Yoshimura, ${ }^{a}$ Martin J. Lear, ${ }^{* a b}$ Isao Ohashi, ${ }^{a}$ Yasuhito Koyama ${ }^{a}$ and Masahiro Hirama ${ }^{* a}$ \\ Received (in Cambridge, UK) 19th April 2007, Accepted 20th June 2007 \\ First published as an Advance Article on the web 29th June 2007 \\ DOI: $10.1039 / b 705932 a$
}

In advanced studies directed toward the total synthesis of the kedarcidin chromophore, we have successfully achieved the late-stage installation of the nine-membered diyne ring in the presence of the highly functionalised ansamacrocyclic bridge.

As highly unstable, complex natural products with unique antiproliferative behaviours, the nine-membered chromophores of the enediyne chromoproteins are most worthy targets of contemporary organic synthesis. ${ }^{1}$ A case in point is the kedarcidin chromophore 1, which possesses an elaborate ansamacrocyclic bridge and two unusual 2-deoxysugar components (Fig. 1). ${ }^{2}$

The major difficulty in synthesising the kedarcidin chromophore 1 pertains to surmounting the high enthalpic and entropic barriers during the construction of the nine-membered, bicyclic core. This difficulty is typically heightened by the short bench-lives of the strained diyne products, whose $\mathrm{C} \equiv \mathrm{C}-\mathrm{C}$ bond-angles are distorted to around $160^{\circ}$. For example, besides the known spontaneous Masamune-Bergman cycloaromatisations of fully-fledged epoxyenediynes, diyne intermediates like 2 readily undergo Cope rearrangements at ambient temperatures (Scheme 1). ${ }^{3}$ Clearly then, the total synthesis of the kedarcidin chromophore demands the late-stage installation of the nine-membered ring (and its continued survival) within a complex chemical environment. In their most recent study, Myers et al. overcame these barriers through an elegant macrocyclic transannulation and have

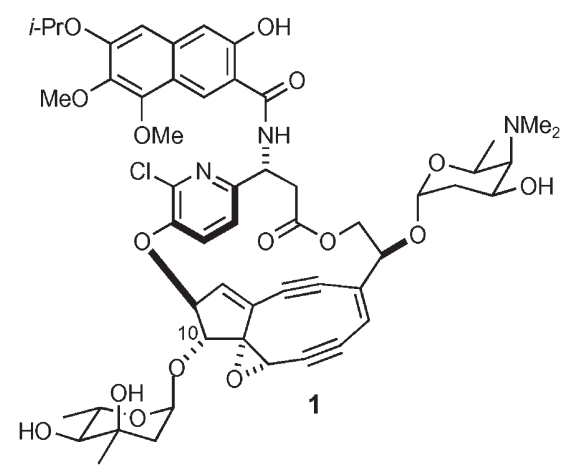

Fig. 1 Structure of kedarcidin chromophore 1 (proposed 1997). ${ }^{2 b}$

${ }^{a}$ Department of Chemistry, Graduate School of Science, Tohoku University, Sendai 980-8578, Japan.

E-mail: hirama@mail.tains.tohoku.ac.jp; Fax: +81-22-795-6566

${ }^{b}$ Department of Chemistry, and Medicinal Chemistry Programme, 3 Science Drive 3, National University of Singapore, Republic of

Singapore 117543. E-mail: chmlmj@nus.edu.sg; Fax: +65-6779-1691

$\uparrow$ Electronic supplementary information (ESI) available: Experimental procedures and characterization data for all new compounds. See DOI: $10.1039 / \mathrm{b} 705932 \mathrm{a}$

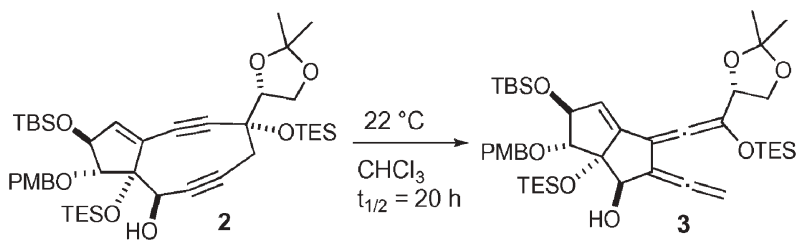

Scheme 1 Decomposition of chromophore model of kedarcidin through a facile Cope rearrangement.

succeeded in the synthesis of $\mathbf{1}$, which has called into question the natural stereochemistry at $\mathrm{C} 10 .{ }^{4}$ In our approach, we have centred on the established success of the $\mathrm{CeCl}_{3} / \mathrm{LiN}(\mathrm{TMS})_{2}$ mediated cyclisation protocol. ${ }^{3}$ We report herein our advanced progress toward the kedarcidin chromophore, which has resulted in the synthesis of the multicyclic ansamacrolide (4).

In our retrosynthesis of $\mathbf{1}$, we elected to use the $\mathrm{CeCl}_{3}$ / LiN(TMS) $)_{2}$-mediated acetylide-aldehyde cyclisation reaction ${ }^{5}$ between $\mathrm{C} 7$ and $\mathrm{C}^{6}$ to form the nine-membered diyne core 4 of the kedarcidin aglycon (Scheme 2). In addition to the concern of the survival of such a complex, highly functionalised system, there was the concern of the resulting stereochemical outcome at $\mathrm{C} 8$ (vide infra).

In order to supply sufficient quantities of the requisite precursor (13) for cyclisation studies, efforts were initially directed at improving our previous synthesis of the ansamacrolide $5^{7}$ These efforts have culminated in the total preparation of $3 \mathrm{~g}$ of the ansamacrolide $\mathbf{5}$ by the development of efficient routes that have enabled the large-scale production of all requisite fragments (see supporting information for the practical synthesis of $\mathbf{5}$ ).

With gram quantities of the ansamacrolide $\mathbf{5}$ in hand, the introduction of the naphthoamide unit to $\mathbf{5}$ was pursued (Scheme 3). After a number of deprotection methods were examined, the best chemoselective procedure to remove the Boc

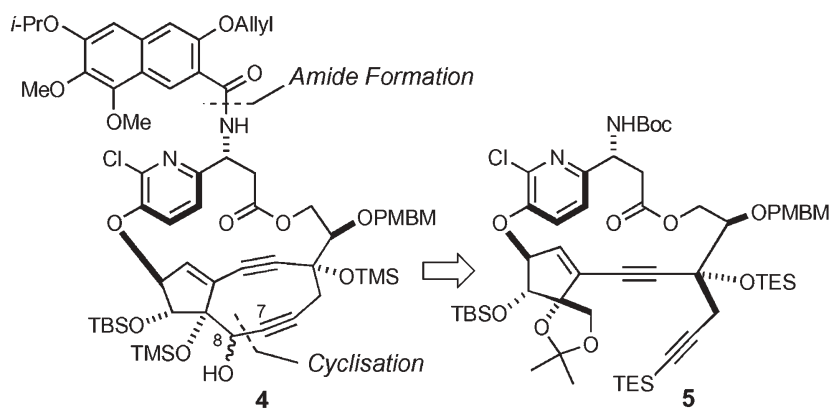

Scheme 2 Synthetic plans. PMBM = p-methoxybenzyloxymethyl. 


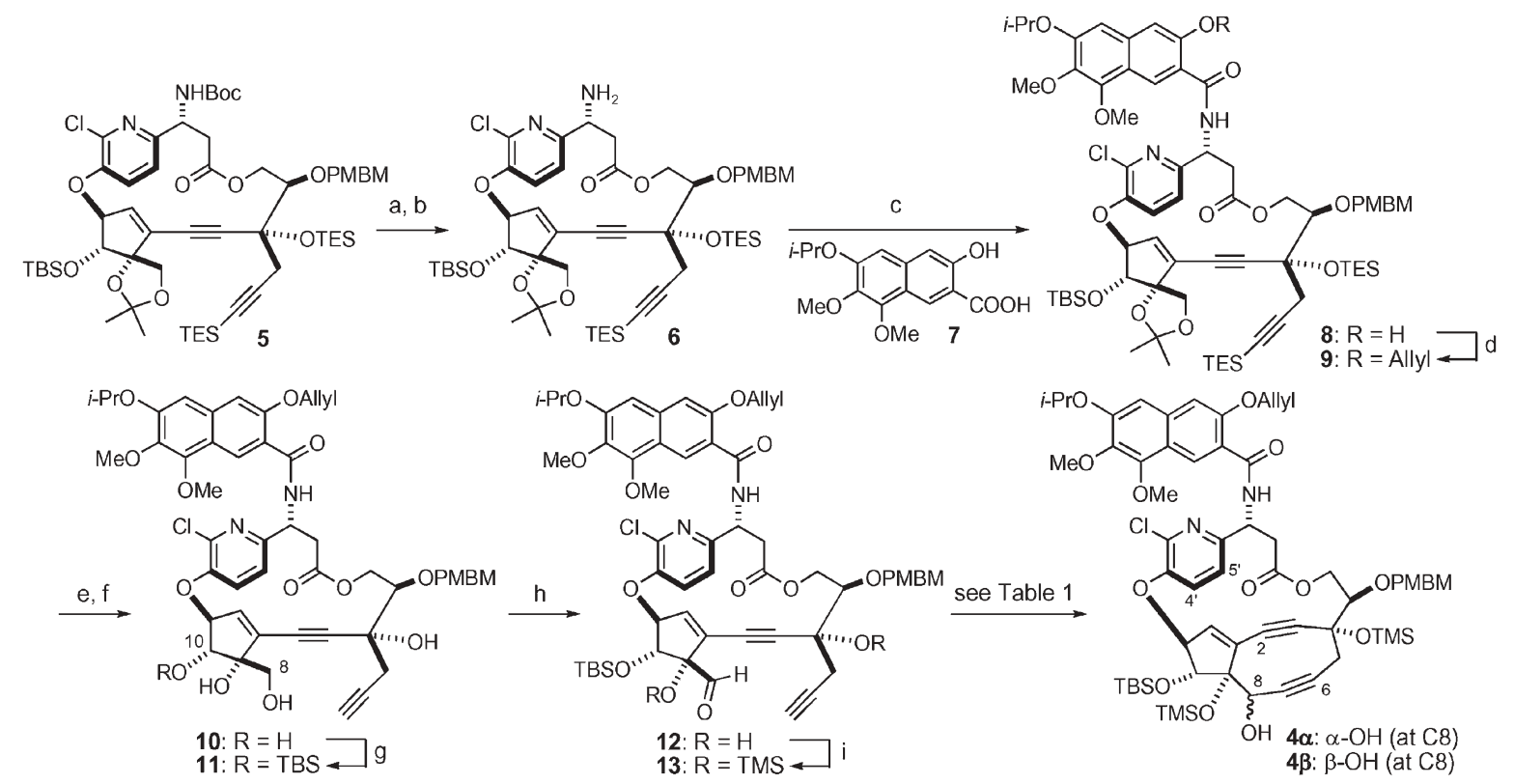

Scheme 3 Reagents and conditions: a) TBSOTf, 2,6-lutidine, $\mathrm{CH}_{2} \mathrm{Cl}_{2},-50$ to $0{ }^{\circ} \mathrm{C}$; b) $\mathrm{SiO}_{2}, \mathrm{CH}_{2} \mathrm{Cl}_{2}$; c) $7, \mathrm{EDC} \cdot \mathrm{HCl}, \mathrm{HOAt} \mathrm{CH}_{2} \mathrm{Cl} 2,0{ }^{\circ} \mathrm{C}(89 \%, 3$ steps); d) allyl bromide, $\mathrm{Cs}_{2} \mathrm{CO}_{3}$, DMF, $0{ }^{\circ} \mathrm{C}(>99 \%)$; e) TBAF, THF, $0{ }^{\circ} \mathrm{C}(93 \%)$; f) TFA-THF- $\mathrm{H}_{2} \mathrm{O}(2: 10: 5), 50{ }^{\circ} \mathrm{C}(71 \%)$; g) TBSCl, Et $3 \mathrm{~N}, \mathrm{DMAP}$, $\mathrm{ClCH}_{2} \mathrm{CH}_{2} \mathrm{Cl}(85 \%)$; h) $\mathrm{SO}_{3}$. pyridine, $\mathrm{Et}_{3} \mathrm{~N}, \mathrm{CH}_{2} \mathrm{Cl}_{2}-\mathrm{DMSO}(10: 3)$ or IBX, MS4A, $\mathrm{CH}_{2} \mathrm{Cl}_{2}-\mathrm{DMSO}(10: 3)$; i) TMSOTf, 2,6-lutidine, $\mathrm{CH}_{2} \mathrm{Cl}_{2},-70{ }^{\circ} \mathrm{C}$.

group in $\mathbf{5}$ first involved transformation to its corresponding $O$-silylcarbamate, as described by Ohfune. ${ }^{8}$ This intermediate was then exposed to silica gel prior to work-up procedures, thereby ensuring the complete liberation of the free amine 6 . Condensation of $\mathbf{6}$ with the naphthoic acid 7 in the presence of $\mathrm{EDC} \cdot \mathrm{HCl}$ and HOAt ${ }^{9}$ resulted in the smooth formation of its amide, which was subsequently protected as its naphtholic allyl ether to afford a total of $2.5 \mathrm{~g}$ of $\mathbf{9}$ in an $88 \%$ four-step yield from $\mathbf{5}$.

At this stage, the selective transformation of the highly oxygenated ansamacrolide 9 to a suitably protected cyclisation precursor was not obvious to us, and the acetonide group in $\mathbf{9}$ was found to be stubborn to deprotection. Eventually, we settled for global desilylation with TBAF followed by hydrolysis of the acetonide by using aqueous TFA at $50{ }^{\circ} \mathrm{C}$ to give the tetraol $\mathbf{1 0}$ (Scheme 3). Quite unexpectedly, treatment of $\mathbf{1 0}$ with TBSCl and $\mathrm{Et}_{3} \mathrm{~N}$ in the presence of a catalytic amount of DMAP gave the triol 11, which had been selectively protected at its C10 secondary alcohol. This outcome was presumably due to the developing transannular steric repulsions that stem from the ansamacrocyclic bridge during the protection of the $\mathrm{C} 8$ primary alcohol.

The search for a reliable oxidation procedure to afford the $\alpha$-hydroxy aldehyde $\mathbf{1 2}$ proved to be an essential pre-requisite to cyclisation studies. Oxidation of $\mathbf{1 1}$ under Dess-Martin or Swern conditions gave complex mixtures, and simplified model systems indicated that either oxidative cleavage of the diol group in $\mathbf{1 1}$ or chlorination of the electron-rich naphthol unit occurred, ${ }^{10}$ respectively. Fortunately, IBX oxidation in the presence of MS4A ${ }^{11}$ afforded 12. This development also led to the oxidation of 11 by $\mathrm{SO}_{3}$.pyridine. Regardless of the method, the requisite cyclisation precursor $\mathbf{1 3}$ was directly formed through the TMS$O$-silylation of $\mathbf{1 2}$, which was subsequently used in crude form. ${ }^{12}$

The ensuing cyclisation of $\mathbf{1 3}$ was first investigated under the standard conditions that had been used to form $2,{ }^{3}$ but the cyclised products $4 \boldsymbol{\alpha}$ and $\mathbf{4 \beta}$ were only isolated in low yield (Table 1,
Table 1 Nine-membered cyclisation study of $\mathbf{1 3}$ (Scheme 3)

\begin{tabular}{llllll}
\hline Entry & Additive & Temp. $\left({ }^{\circ} \mathrm{C}\right)$ & Time $(\mathrm{h})$ & Yield $(\%)$ & $\mathbf{4 \alpha / 4 \beta ^ { b }}$ \\
\hline 1 & $\mathrm{CeCl}_{3}$ & -25 to rt & 1 & $<7$ & $2 / 1$ \\
2 & $\mathrm{CeCl}_{3}$ & -15 & 18 & 26 & $3 / 1$ \\
3 & $\mathrm{CeCl}_{3}$ & -25 & 25 & 47 & $3 / 1$ \\
4 & $\mathrm{CeCl}_{3}$ & -50 & 69 & 12 & $3 / 1$ \\
5 & none & -25 & 36 & 0 & - \\
6 & $\mathrm{YbCl}_{3}$ & -25 & 36 & 22 & $2 / 3$
\end{tabular}

${ }^{a}$ Reactions performed by adding 13 to $30-50$ equivalents of premixed $\mathrm{CeCl}_{3}$ (or $\mathrm{YbCl}_{3}$ )-LiN(TMS) ${ }_{2}$ [100 : 95] in THF [1 mM]; combined yields relate to $\mathbf{4} \boldsymbol{\alpha} / \boldsymbol{\beta}$ over 3 steps from $\mathbf{1 1}$, see Scheme 3 . ${ }^{b}$ Ratios determined on crude by ${ }^{1} \mathrm{H}-\mathrm{NMR}$ analysis $(500 \mathrm{MHz})$.

entry 1). The reaction temperature affected both the yield and the stereochemical outcome, and the best results were achieved at $-25{ }^{\circ} \mathrm{C}$ to produce the cyclised products $\mathbf{4} \boldsymbol{\alpha}$ and $\mathbf{4 \beta}$ in a $47 \%$ combined yield, over three steps from 11 (Table 1, entries 2-4). In contrast to the cyclisation of previous model systems, such as that to afford $\mathbf{2},{ }^{3}$ the cyclisation of $\mathbf{1 3}$ displayed an $\alpha$-stereoselectivity at the $\mathrm{C}-8$ alcohol ( $c f . \mathbf{4} \boldsymbol{\alpha})$, presumably due to the steric repulsion of the ansamacrolide framework ( $c f$. Scheme 4). Addition of $\mathrm{CeCl}_{3}$

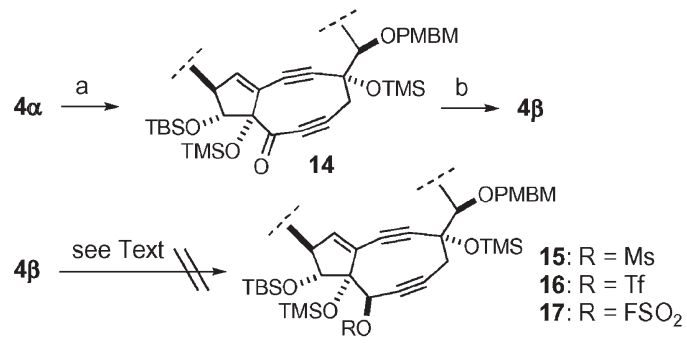

Scheme 4 Attempted sulfonate formation of $4 \beta$. Reagents and conditions: a) Dess-Martin periodinane, $\mathrm{NaHCO}_{3}, \mathrm{CH}_{2} \mathrm{Cl}_{2}(93 \%)$; b) $\mathrm{Zn}\left(\mathrm{BH}_{4}\right)_{2}$, $\mathrm{Et}_{2} \mathrm{O},-30{ }^{\circ} \mathrm{C}(57 \%)$. 
was indispensable for this reaction (Table 1, entry 5). Interestingly, $\mathrm{CeCl}_{3}$ when replaced by $\mathrm{YbCl}_{3}$, ${ }^{13}$ reversed the stereoselectivity to give $\mathbf{4} \boldsymbol{\beta}$ predominantly (Table 1 , entry 6 ). Although $\mathbf{4 \alpha}$ was not stable at room temperature (half-life was approximately $13 \mathrm{~h}$ in $\left.\mathrm{C}_{6} \mathrm{D}_{6}\right), 4 \alpha$ could be stored in a benzene matrix at $-30{ }^{\circ} \mathrm{C}$ for one week without deterioration. The $\mathrm{C} 8$-stereochemistry in $\mathbf{4} \boldsymbol{\alpha}$ was determined unambiguously by the NOE study of its mesylate (see compound 18 in supporting information; definitive NOEs between $\mathrm{H} 8$ and $\mathrm{H}^{\prime}$; $\mathrm{H} 8$ and $\mathrm{H}^{\prime}$ ). Moreover, the downfield-shift of key ${ }^{13} \mathrm{C}-\mathrm{NMR}$ data of $\mathbf{4} \boldsymbol{\alpha}$ revealed that the alkyne carbons $(\mathrm{C} 2 / 3, \mathrm{C} 6 / 7)$ are under significant strain.

In our earlier study, we succeeded in the epoxide formation from $\beta$-alcohol at the C8-position of $\mathbf{2}$ via mesylation followed by treatment with TBAF. ${ }^{3 b}$ The conversion of the alcohol at $\mathrm{C} 8$ of the major cyclised product $\mathbf{4} \boldsymbol{\alpha}$ to a suitable leaving group is thus key to this transformation. Inversion of the secondary alcohol of $\mathbf{4} \boldsymbol{\alpha}$ was planned to be carried out via an oxidation-reduction sequence (Scheme 4). After a number of investigations, $\mathbf{4} \boldsymbol{\alpha}$ was oxidised to the ynone 14 by using Dess-Martin periodinane in the presence of $\mathrm{NaHCO}_{3}$. The chemoselective reduction of $\mathbf{1 4}$ was then examined. The best reducing conditions involved freshly prepared $\mathrm{Zn}\left(\mathrm{BH}_{4}\right)_{2}$ in $\mathrm{Et}_{2} \mathrm{O}$ at $-30{ }^{\circ} \mathrm{C}$, producing the $\beta$-alcohol $\mathbf{4 \beta}$ in $57 \%$ yield. Other reductants such as $\mathrm{NaBH}_{4}-\mathrm{CeCl}_{3} \cdot 7 \mathrm{H}_{2} \mathrm{O}$ or $\mathrm{LiB}(\mathrm{sec}-\mathrm{Bu})_{3} \mathrm{H}$ gave complex mixtures, including the formation of TMS deprotected products and over-reduced products.

Having developed suitable conditions for the inversion of the secondary alcohol of $\mathbf{4 \alpha}$, the transformation of the alcohol of $\mathbf{4 \beta}$ to a suitable leaving group was investigated (Scheme 4). Mesylate formation of $\boldsymbol{4} \boldsymbol{\beta}$ under various conditions, such as $\mathrm{MsCl}-\mathrm{Et}_{3} \mathrm{~N}$, $\mathrm{MsCl}-\mathrm{DMAP}, \mathrm{MsCl}-\mathrm{Me}_{2} \mathrm{~N}\left(\mathrm{CH}_{2}\right)_{6} \mathrm{NMe}_{2}{ }^{14}$ or $\mathrm{MsCl}-\mathrm{AgO}-\mathrm{KI}^{15}$ did not give any mesylate $15 .{ }^{16} \mathrm{Next}$, triflate formation of $\mathbf{4 \beta}$ was examined. However, triflate formation of $\mathbf{4 \beta}$ by using $\mathrm{Tf}_{2} \mathrm{O}^{17}$ in the presence of various bases such as 2,6-di-t-butylpyridine, 2,6lutidine or pyridine, and $\mathrm{TfCl}-\mathrm{Et}_{3} \mathrm{~N}^{16}$ was unsuccessful. Fluorosulfonate formation of $\mathbf{4} \boldsymbol{\beta}$ by using $\mathrm{FSO}_{2} \mathrm{Cl}^{18}$ which would act as a small sulfonation reagent, was also unsuccessful.

On the basis of these results, it is clear that our failure in forming the mesylate, triflate, or fluorosulfonate forms of $\mathbf{4 \beta}$ is attributable to steric repulsions of the ansa-bridge. An energy minimized model structure (MM2* Macromodel ver. 6.0) of the simplified $\beta$-alcohol compound showed that the alcohol at $\mathrm{C} 8$ is screened by the ansa-bridge giving a small transannular cavity (see supporting information). It seems, therefore, that an alternative synthetic strategy is required for the construction of the nine-membered epoxy diyne core of $\mathbf{1}$.

To conclude, we have succeeded in constructing the multicyclic diyne ansamacrolide $(\mathbf{4} \boldsymbol{\alpha} / \boldsymbol{\beta})$, possessing the entire carbon skeleton of the kedarcidin chromophore aglycon through the remarkable facility of $\mathrm{CeCl}_{3}$ to moderate the anionic formation of unstable, nine-membered cores. We have demonstrated $\mathrm{YbCl}_{3}$ to be an interesting alternative to $\mathrm{CeCl}_{3}$ that can better accommodate the sterically imposing, macrocyclic framework of $\mathbf{4}$. It should also be noted that the final three-step sequence has been reliably performed over 15 times to give $\mathbf{4} \boldsymbol{\alpha} / \boldsymbol{\beta}$ in yields of $38-47 \%$ (starting from $30-90 \mathrm{mg}$ of 11). In the meantime, we are addressing several stereochemical issues ${ }^{4,19}$ and are striving to complete our endeavours towards the total synthesis of the kedarcidin chromophore.

This work was supported under the CREST program from JST. A Fellowship to F.Y. from the Japanese Society for the Promotion of Science for Young Scientists is gratefully acknowledged. The authors wish to thank Dr Masako Ueno, Mr Kazuo Sasaki, Mr Takeyoshi Kondo and Mr Toshio Sato (Research and Analysis Center for Giant Molecules, Graduate School of Science, Tohoku University) for mass spectral and NMR analysis.

\section{Notes and references}

1 Reviews: (a) J. W. Grissonm, G. U. Gunawardena, D. Klingberg and D. Huang, Tetrahedron, 1996, 52, 6453; (b) S. J. Danishefsky and M. D. Shair, J. Org. Chem., 1996, 61, 16.

2 Isolation and proposed structure of 1: (a) J. E. Leet, D. R. Schroeder, D. R. Langley, K. L. Colson, S. Huang, K. E. Klohr, M. S. Lee, J. Golik, S. J. Hofstead, T. W. Doyle and J. A. Matson, J. Am. Chem. Soc., 1992, 114, 8432. Structure revision: (b) S. Kawata, A. Ashizawa and M. Hirama, J. Am. Chem. Soc., 1997, 119, 12012.

3 (a) K. Iida and M. Hirama, J. Am. Chem. Soc., 1994, 116, 10310; (b) S. Kawata, F. Yoshimura, J. Irie, H. Ehara and M. Hirama, Synlett, 1997, 250

4 Synthesis of 1 and stereochemical revision of the kedarcidin chromophore: F. Ren, P. C. Hogan, A. J. Anderson and A. G. Myers, J. Am. Chem. Soc., in press.

5 Effect of $\mathrm{CeCl}_{3}$ on acetylide-aldehyde additions: (a) A. G. Myers, P. M. Harrington and E. Y. Kuo, J. Am. Chem. Soc., 1991, 113, 694 (b) T. Nishikawa, M. Isobe and T. Goto, Synlett, 1991, 393.

6 The carbon numbering follows that for the kedarcidin chromophore $(\mathbf{1})^{2}$.

7 F. Yoshimura, S. Kawata and M. Hirama, Tetrahedron Lett., 1999, 40, 8281.

8 M. Sakaitani and Y. Ohfune, J. Org. Chem., 1990, 55, 870.

9 L. A. Carpino, J. Am. Chem. Soc., 1993, 115, 4397.

10 Aromatic-chlorinations with $\left[\mathrm{Me}_{2} \mathrm{~S}^{+} \mathrm{Cl}_{1} \mathrm{Cl}^{-}\right.$: G. A. Olah, L. Ohannesian and M. Arvanaghi, Synthesis, 1986, 868.

11 M. Frigerio and M. Santagostino, Tetrahedron Lett., 1994, 35, 8019. Addition of MS4A is required to suppress hydrate formation.

12 Compounds $\mathbf{1 2}$ and $\mathbf{1 3}$ are unstable to silica gel and should be used immediately in the optimised three-step sequence from 13 to 4 .

13 This reversion in stereoselectivity was anticipated on the basis of the smaller ionic radius of $\mathrm{Yb}^{3+}$ as compared to $\mathrm{Ce}^{3+}$ (R. D. Shannon, Acta Crystallogr., Sect. A: Cryst. Phys., Diffr., Theor. Gen. Cryst., 1976, 32, 751) which would incur less steric interactions from the ansamacrocyclic bridge during a nine-membered cyclisation, also see: K. Utimoto, A. Nakamura and S. Matsubara, J. Am. Chem. Soc., 1990, 112, 8189; D. Enders and J. Tiebes, Liebigs Ann. Chem., 1993, 173; S. Matsubara, T. Ikeda, K. Oshima and K. Utimoto, Chem. Lett., 2001, 1226.

14 Y. Yoshida, K. Shimonishi, Y. Sakakura, S. Okada, N. Aso and Y. Tanabe, Synthesis, 1999, 1633.

15 A. Bouzide, N. LeBerre and G. Sauvé, Tetrahedron Lett., 2001, 42, 8781.

$164 \beta$ was recovered.

17 A simplified model study indicated that $\mathrm{Tf}_{2} \mathrm{O}$-induced imidazo[1,5a]pyridine formation likely occurred instead of triflate formation, see the formation of $\mathbf{2 3}$ from $\mathbf{2 2}$ in supporting information.

18 V. P. Reddy, D. R. Bellew and G. K. S. Prakash, J. Fluorine Chem., 1992, 46, 165.

19 Y. Koyama, M. J. Lear, F. Yoshimura, I. Ohashi, T. Mashimo and M. Hirama, Org. Lett., 2005, 7, 267. 